\title{
Asymptotic and periodic motion around collinear equilibria in Chermnykh's problem
}

\author{
E. A. Perdios ${ }^{1}$ and O. Ragos ${ }^{2}$ \\ ${ }^{1}$ Department of Engineering Sciences, University of Patras, 26500 Patras, Greece \\ 2 Department of Mathematics, University of Patras, 26500 Patras, Greece \\ Received 21 February 2003 / Accepted 29 September 2003

\begin{abstract}
In this paper we study the asymptotic motion to the collinear equilibrium points of Chermnykh's problem. More these solutions are closely connected to the families of periodic orbits generated around these equilibria.
\end{abstract} \\ specifically, we give three kinds of non-symmetric doubly-asymptotic solutions emanating from $L_{1}$ and $L_{3}$. We also show that
}

Key words. celestial mechanics - methods: numerical

\section{Introduction}

An interesting case of motion in dynamical systems is when moving bodies remain for a long time in the vicinity of unstable equilibrium points of these systems. Such a situation may be due to the fact that the bodies trace orbits which asymptotically terminate on these points. Several studies of this kind of motion have been presented up to now. Asymptotic orbits to equilibrium points were first studied by Strömgren (1935) in the case of the classical restricted three-body problem. These orbits were spiraling asymptotically to the triangular equilibrium points and were connected to orbits of termination of families of periodic solutions. Non-spiraling asymptotic orbits to the collinear equilibrium points were studied by Deprit \& Henrard (1965). There, the existence of nonsymmetric asymptotic orbits to $L_{3}$ were also discussed having images with respect to the axis that contains the two primaries. Homoclinic and heteroclinic asymptotic orbits to the collinear equilibrium points have also been studied numerically for the above problem by Danby (1967), Markellos (1991), and Perdios \& Markellos (1990), while Llibre et al. (1985) and Koon et al. (2000) give a detailed analytical and numerical study. Also, Perdios (1996, 1997) and Perdios et al. (2001) have worked on symmetric asymptotic motion to the collinear libration points of the photogravitational restricted three-body problem. Some results concerning doubly asymptotic orbits to the collinear points in the elliptical restricted problem have been presented by Perdios (1983). A numerical study of doubly asymptotic solutions to the collinear libration points of the general gravitational three-body problem is given by Perdios \& Kalantonis (2002).

In this paper we compute non-symmetric asymptotic orbits to the collinear equilibrium points and discuss their relation to

Send offprint requests to: E. A. Perdios,

e-mail: perdios@des.upatras.gr families of symmetric periodic solutions in Chermnykh's problem (Chermnykh 1987). These orbits connect a collinear equilibrium point with the vicinity of one of the triangular points. We also show that, starting from these asymptotic orbits, we can find neighbor periodic solutions extracted from them. We have computed these orbits as well as the corresponding families containing them for two example cases. One of these families ends asymptotically in a periodic orbit around $L_{3}$, while the other one terminates asymptotically in a periodic orbit around the inner collinear equilibrium point, and spiraling asymptotically at $L_{4}$.

The paper is organized as follows. In the next section we describe Chermnykh's problem. In Sect. 3, we study the position and the stability of the equilibrium points of the modelproblem. In Sect. 4, we give a fourth order approximation of the solution around the collinear equilibrium points for the determination of the relevant outgoing and incoming eigenvectors. In Sect. 5, we describe the procedure employed to calculate non-symmetric doubly asymptotic orbits as well as how to extract the corresponding neighbor symmetric periodic solutions. Finally, in the same section, we give the numerical results obtained by our analysis.

\section{Equations of motion}

Chermnykh's problem is a generalization of the circular plane restricted three-body problem and Euler's problem of two fixed gravitational centers; it can be used to model several dynamical systems appearing in Celestial Mechanics and Chemistry. This problem concerns the motion of a particle having infinitesimal mass in the orbital plane of a dumb-bell which is rotating with constant angular velocity $\omega$ around its mass center (for details, see Chermnykh 1987; Gożdziewski \& Maciejewski 1998). To study this problem we consider a barycentric canonical system of coordinates whose $\mathrm{O} x_{1}$ axis always contains the two 
primaries. The constant distance between these primaries and the sum of their masses are considered to be equal to unity, while $\omega \in[0, \infty)$. If we denote by $1-\mu$ and $\mu, \mu \leq 1 / 2$, the masses $m_{1}$ and $m_{2}$ of the primaries, the motion of the third particle is described by the system:

$$
\begin{aligned}
& \dot{x}_{1}=x_{3}, \\
& \dot{x}_{2}=x_{4}, \\
& \dot{x}_{3}=2 \omega x_{4}+\left(\omega^{2}-Q\right) x_{1}-\mu(1-\mu) R, \\
& \dot{x}_{4}=-2 \omega x_{3}+\left(\omega^{2}-Q\right) x_{2},
\end{aligned}
$$

where

$$
\begin{array}{ll}
R=\frac{1}{r_{1}^{3}}-\frac{1}{r_{2}^{3}}, & Q=\frac{1-\mu}{r_{1}^{3}}+\frac{\mu}{r_{2}^{3}}, \\
r_{1}=\sqrt{\left(x_{1}+\mu\right)^{2}+x_{2}^{2}}, & r_{2}=\sqrt{\left(x_{1}+\mu-1\right)^{2}+x_{2}^{2}} .
\end{array}
$$

The Jacobian integral of this system is given by:

$J=\left(x_{3}^{2}+x_{4}^{2}\right)-\omega^{2}\left(x_{1}^{2}+x_{2}^{2}\right)-\frac{2(1-\mu)}{r_{1}}-\frac{2 \mu}{r_{2}}=C$.

Note that, for $\omega=1$, we get the classical circular restricted plane three-body problem, while, for $\omega=0$, Euler's problem of two fixed gravitational centers is obtained.

\section{Position and stability of equilibrium points}

In Chermnykh's problem there are two kinds of equilibrium points. The points of the first kind are collinear to the $\mathrm{O} x_{1}$ axis. Their positions are determined by solving the equations:

$$
\left(\omega^{2}-Q\right) x_{1}-\mu(1-\mu) R=0, \quad x_{2}=0
$$

A numerical investigation shows that there are three such points for each set of values of $\mu$ and $\omega$. These points are named $L_{1}$, $L_{2}$ and $L_{3}$. The position of the point $L_{1}$ belongs to the interval $(-\mu, 1-\mu)$; it tends to $1-\mu$ as $\omega$ tends to 0 , and to $-\mu$ as $\omega$ tends to $+\infty$. The position of $L_{2}$ belongs to $(1-\mu,+\infty)$; it tends to $+\infty$ as $\omega$ tends to 0 , and to $1-\mu$ as $\omega$ tends to $+\infty$. The position of $L_{3}$ belongs to $(-\infty,-\mu)$; it tends to $-\infty$ as $\omega$ tends to 0 , and to $-\mu$ as $\omega$ tends to $+\infty$.

The equilibrium points of the second kind are called triangular and they exist for $\omega \in(0,2 \sqrt{2})$. Their positions are symmetrical with respect to the $\mathrm{O} x_{1}$ axis:

$$
x_{1}=\frac{1-2 \mu}{2}, \quad x_{2}= \pm \sqrt{\omega^{-4 / 3}-\frac{1}{4}}
$$

These equilibrium points are named, as usual, $L_{4}$ and $L_{5}$, respectively. As the parameter $\omega$ tends to 0 they escape to infinity, while in the limiting case $\omega \rightarrow 2 \sqrt{2}$ they tend to coincide with $L_{1}$ (Gożdziewski \& Maciejewski 1998).

In order to study the linear stability of any equilibrium point $L$, we transfer the origin of the coordinate system to its position $\left(x_{1 L}, x_{2 L}\right)$ by means of:

$x_{1}=x_{1 L}+\xi_{1}, \quad x_{2}=x_{2 L}+\xi_{2}$,

and, then, linearize (1). The linearized system can be written as follows:

$\left(\dot{\xi}_{1}, \dot{\xi}_{2}, \dot{\xi}_{3}, \dot{\xi}_{4}\right)^{T}=\mathcal{M} \cdot\left(\xi_{1}, \xi_{2}, \xi_{3}, \xi_{4}\right)^{T}$ where

$\mathcal{M}=\left(\begin{array}{cccc}0 & 0 & 1 & 0 \\ 0 & 0 & 0 & 1 \\ A_{1}^{*} & A_{2}^{*} & 0 & 2 \omega \\ B_{1}^{*} & B_{2}^{*} & -2 \omega & 0\end{array}\right)$.

$A_{i}^{*}, B_{i}^{*}, i=1,2$, are expressions which depend on $\mu, \omega$ and the position of the equilibrium point.

For the collinear libration points, these expressions are:

$A_{1}^{*}=\omega^{2}-Q_{0}^{*}-x_{1 L} Q_{1}^{*}-\mu(1-\mu) R_{1}^{*}$,

$A_{2}^{*}=0, \quad B_{1}^{*}=0, \quad B_{2}^{*}=\omega^{2}-Q_{0}^{*}$,

where

$Q_{0}^{*}=\frac{1-\mu}{r_{1_{L}}^{3}}+\frac{\mu}{r_{2 L}^{3}}$,

$Q_{1}^{*}=-\frac{3(1-\mu)\left(x_{1 L}+\mu\right)}{r_{1_{L}}^{5}}-\frac{3 \mu\left(x_{1 L}+\mu-1\right)}{r_{2_{L}}^{5}}$,

$R_{1}^{*}=-\frac{3\left(x_{1 L}+\mu\right)}{r_{1_{L}}^{5}}+\frac{3\left(x_{1 L}+\mu-1\right)}{r_{2_{L}}^{5}}$,

and $r_{1 L}=\left|x_{1 L}+\mu\right|$ and $r_{2 L}=\left|x_{1 L}+\mu-1\right|$. The characteristic equation of $\mathcal{M}$ is, in this case:

$\lambda^{4}+\left(4 \omega^{2}-A_{1}^{*}-B_{2}^{*}\right) \lambda^{2}+A_{1}^{*} B_{2}^{*}=0$,

while the corresponding eigenvalues are:

$\lambda_{1,2,3,4}= \pm \sqrt{\frac{1}{2}\left(\gamma \pm \sqrt{\gamma^{2}-4 A_{1}^{*} B_{2}^{*}}\right)}$

where $\gamma=A_{1}^{*}+B_{2}^{*}-4 \omega^{2}$. A numerical study of these eigenvalues for the whole range of $\mu$ and $\omega$ leads to the conclusion that the collinear equilibrium points are always unstable.

In the case of the triangular points, the elements of $\mathcal{M}$ are:

$A_{1}^{*}=\omega^{2}-Q_{0}^{*}-x_{1 L} Q_{1}^{*}-\mu(1-\mu) R_{1}^{*}$,

$A_{2}^{*}=-x_{1 L} Q_{2}^{*}-\mu(1-\mu) R_{2}^{*}$,

$B_{1}^{*}=A_{2}^{*}, \quad B_{2}^{*}=\omega^{2}-Q_{0}^{*}-Q_{2}^{*} x_{2 L}$.

The expressions $Q_{0}^{*}, Q_{1}^{*}$ and $R_{1}^{*}$ are given by (4) while

$Q_{2}^{*}=-\frac{3(1-\mu) x_{2 L}}{r_{1_{L}}^{5}}-\frac{3 \mu x_{2 L}}{r_{2_{L}}^{5}}, R_{2}^{*}=-\frac{3 x_{2 L}}{r_{1_{L}}^{5}}+\frac{3 x_{2 L}}{r_{2_{L}}^{5}}$,

where $r_{1 L}=\sqrt{\left(x_{1 L}+\mu\right)^{2}+x_{2 L}^{2}}$ and $r_{2 L}=$ $\sqrt{\left(x_{1 L}+\mu-1\right)^{2}+x_{2 L}^{2}}$. The characteristic equation of $\mathcal{M}$ is, in this case:

$\lambda^{4}+\left(4 \omega^{2}-A_{1}^{*}-B_{2}^{*}\right) \lambda^{2}+A_{1}^{*} B_{2}^{*}-A_{2}^{* 2}=0$,

while its eigenvalues are:

$\lambda_{1,2,3,4}= \pm \sqrt{\frac{1}{2}\left(\gamma \pm \sqrt{\gamma^{2}-4\left(A_{1}^{*} B_{2}^{*}-A_{2}^{* 2}\right)}\right)}$.

By studying the characteristic equation, it can be found that $L_{4}$ and $L_{5}$ are stable under the condition:

$1-9 \mu(1-\mu) \omega^{8 / 3}\left(4 \omega^{-4 / 3}-1\right)>0$.

A detailed discussion of the linear and nonlinear stability of the triangular equilibrium points is given in Gożdziewski \& Maciejewski (1998). 


\section{Asymptotic motion to the collinear equilibrium points}

To study the motion in the near vicinity of any collinear equilibrium point $L$, we first apply the transformation (2) to system (1) and, then, expand the right hand side of the resulting system in Taylor series around $\left(x_{1 L}, 0\right)$. An approximation of this system including all terms up to the fourth order, is given by:

$$
\begin{aligned}
\ddot{\xi}_{1}-2 \omega \dot{\xi}_{2}= & A_{1} \xi_{1}+A_{2} \xi_{1}^{2}+A_{3} \xi_{2}^{2}+A_{4} \xi_{1} \xi_{2}^{2} \\
& +A_{5} \xi_{1}^{3}+A_{6} \xi_{1}^{4}+A_{7} \xi_{2}^{4}+A_{8} \xi_{1}^{2} \xi_{2}^{2}, \\
\ddot{\xi}_{2}+2 \omega \dot{\xi}_{1}= & B_{1} \xi_{2}+B_{2} \xi_{1} \xi_{2}+B_{3} \xi_{1}^{2} \xi_{2}+B_{4} \xi_{2}^{3} \\
& +B_{5} \xi_{1} \xi_{2}^{3}+B_{6} \xi_{1}^{3} \xi_{2},
\end{aligned}
$$

where we have abbreviated:

$$
\begin{aligned}
& A_{0}=x_{1 L}\left(\omega^{2}-Q_{0}\right)-\mu(1-\mu) R_{0}, \\
& A_{1}=\omega^{2}-Q_{0}-x_{1 L} Q_{1}-\mu(1-\mu) R_{1}, \\
& A_{2}=-Q_{1}-4 A_{3}, \\
& A_{3}=x_{1 L} Q_{2}+\mu(1-\mu) R_{2}, \\
& A_{4}=Q_{2}-3\left(x_{1 L} Q_{3}+\mu(1-\mu) R_{3}\right), \\
& A_{5}=4\left(x_{1 L} Q_{3}-Q_{2}+\mu(1-\mu) R_{3}\right), \\
& A_{6}=4\left(Q_{3}+2 A_{7}\right), \\
& A_{7}=-x_{1 L} Q_{4}-\mu(1-\mu) R_{4}, \\
& A_{8}=-3\left(Q_{3}+4 A_{7}\right), \\
& B_{1}=\omega^{2}-Q_{0}, \quad B_{2}=-Q_{1}, \quad B_{3}=-4 Q_{2} \\
& B_{4}=Q_{2}, \quad B_{5}=-3 Q_{3}, \quad B_{6}=4 Q_{3},
\end{aligned}
$$

with

$Q_{0}=\frac{(1-\mu)}{|a|^{3}}+\frac{\mu}{|a-1|^{3}}, \quad R_{0}=\frac{1}{|a|^{3}}-\frac{1}{|a-1|^{3}}$,

$Q_{1}=-\frac{3(1-\mu)}{a|a|^{3}}-\frac{3 \mu}{(a-1)|a-1|^{3}}$,

$R_{1}=-\frac{3}{a|a|^{3}}+\frac{3}{(a-1)|a-1|^{3}}$,

$Q_{2}=\frac{3(1-\mu)}{2|a|^{5}}+\frac{3 \mu}{2|a-1|^{5}}, \quad R_{2}=\frac{3}{2|a|^{5}}-\frac{3}{2|a-1|^{5}}$,

$Q_{3}=\frac{5(1-\mu)}{2 a|a|^{5}}+\frac{5 \mu}{2(a-1)|a-1|^{5}}$,

$R_{3}=\frac{5}{2 a|a|^{5}}-\frac{5}{2(a-1)|a-1|^{5}}$,

$Q_{4}=\frac{15(1-\mu)}{8|a|^{7}}+\frac{15 \mu}{8|a-1|^{7}}, \quad R_{4}=\frac{15}{8|a|^{7}}-\frac{15}{8|a-1|^{7}}$,

and $a=x_{1 L}+\mu$.

By using a classical method (see Deprit \& Henrard 1965), we express the solution of system (11) in terms of a small orbital parameter $\epsilon$ :

$\xi_{1}(t)=\sum_{i=1}^{4} \xi_{1 i}(t) \epsilon^{i}, \quad \xi_{2}(t)=\sum_{i=1}^{4} \xi_{2 i}(t) \epsilon^{i}$

The parameter $\epsilon$ provides a measure of the distance from the equilibrium along the unstable manifold. For sufficiently small $\epsilon$, the fourth-order expansion provides sufficient accuracy for the solution. The accuracy obtained can be tested to determine the appropriate size for $\epsilon$ by backward integration (see below). If this solution is replaced in (11) and the coefficients of the corresponding terms are equated, we have that:

$\ddot{\xi}_{11}-2 \omega \dot{\xi}_{21}=A_{1} \xi_{11}$

$\ddot{\xi}_{21}+2 \omega \dot{\xi}_{11}=B_{1} \xi_{21}$,

$\ddot{\xi}_{12}-2 \omega \dot{\xi}_{22}=A_{1} \xi_{12}+A_{2} \xi_{11}^{2}+A_{3} \xi_{21}^{2}$,

$\ddot{\xi}_{22}+2 \omega \dot{\xi}_{12}=B_{1} \xi_{22}+B_{2} \xi_{11} \xi_{21}$,

$\ddot{\xi}_{13}-2 \omega \dot{\xi}_{23}=A_{1} \xi_{13}+2 A_{2} \xi_{11} \xi_{12}+2 A_{3} \xi_{21} \xi_{22}$

$+A_{4} \xi_{11} \xi_{21}^{2}+A_{5} \xi_{11}^{3}$

$\ddot{\xi}_{23}+2 \omega \dot{\xi}_{13}=B_{1} \xi_{23}+B_{2} \xi_{11} \xi_{22}+B_{2} \xi_{12} \xi_{21}$

$$
+B_{3} \xi_{11}^{2} \xi_{21}+B_{4} \xi_{21}^{3}
$$

$$
\begin{aligned}
\ddot{\xi}_{14}-2 \omega \dot{\xi}_{24}= & A_{1} \xi_{14}+A_{2} \xi_{12}^{2}+2 A_{2} \xi_{11} \xi_{13}+A_{3} \xi_{22}^{2} \\
& +2 A_{3} \xi_{21} \xi_{23}+A_{4} \xi_{12} \xi_{21}^{2} \\
& +2 A_{4} \xi_{11} \xi_{21} \xi_{22}+3 A_{5} \xi_{11}^{2} \xi_{12} \\
& +A_{6} \xi_{11}^{4}+A_{7} \xi_{21}^{4}+A_{8} \xi_{11}^{2} \xi_{21}^{2} \\
\ddot{\xi}_{24}+2 \omega \dot{\xi}_{14}= & B_{1} \xi_{24}+B_{2} \xi_{12} \xi_{22}+B_{2} \xi_{11} \xi_{23} \\
& +B_{2} \xi_{13} \xi_{21}+2 B_{3} \xi_{11} \xi_{12} \xi_{21} \\
& +B_{3} \xi_{11}^{2} \xi_{22}+3 B_{4} \xi_{21}^{2} \xi_{22} \\
& +B_{5} \xi_{11} \xi_{21}^{3}+B_{6} \xi_{11}^{3} \xi_{21}
\end{aligned}
$$

For the determination of a doubly asymptotic orbit, we use the particular solutions of systems (13)-(16) along the eigenvector which corresponds to the largest positive eigenvalue of the matrix $\mathcal{M}$. These solutions are of the form:

$\xi_{k}(t)=h_{k} \mathrm{e}^{k \lambda_{1}}, \quad \eta_{k}(t)=g_{k} \mathrm{e}^{k \lambda_{1}}, \quad k=1,2,3,4$,

where $\lambda_{1}$ is the above mentioned eigenvalue. This can be easily verified. By replacing (17) in (13) we find that:

$h_{1}=1, \quad g_{1}=\frac{\lambda_{1}^{2}-A_{1}}{2 \omega \lambda_{1}}=\frac{2 \omega \lambda_{1}}{B_{1}-\lambda_{1}^{2}}$.

Then,

$h_{2}=\frac{4 \omega B_{2} g_{1} \lambda_{1}+\left(4 \lambda_{1}^{2}-B_{1}\right) \Phi_{0}}{16 \lambda_{1}^{4}+4\left(4 \omega^{2}-A_{1}-B_{1}\right) \lambda_{1}^{2}+A_{1} B_{1}}$,
$h_{3}=\frac{\left(9 \lambda_{1}^{2}-B_{1}\right) \Phi_{1}+6 \omega \lambda_{1} \Phi_{2}}{81 \lambda_{1}^{4}+9\left(4 \omega^{2}-A_{1}-B_{1}\right) \lambda_{1}^{2}+A_{1} B_{1}}$,

$h_{4}=\frac{\left(16 \lambda_{1}^{2}-B_{1}\right) \Phi_{3}+8 \omega \lambda_{1} \Phi_{4}}{256 \lambda_{1}^{4}+16\left(4 \omega^{2}-A_{1}-B_{1}\right) \lambda_{1}^{2}+A_{1} B_{1}}$,

$g_{2}=\frac{\left(4 \lambda_{1}^{2}-A_{1}\right) B_{2} g_{1}-4 \omega \Phi_{0} \lambda_{1}}{16 \lambda_{1}^{4}+4\left(4 \omega^{2}-A_{1}-B_{1}\right) \lambda_{1}^{2}+A_{1} B_{1}}$,

$g_{3}=\frac{\left(9 \lambda_{1}^{2}-A_{1}\right) \Phi_{2}-6 \omega \lambda_{1} \Phi_{1}}{81 \lambda_{1}^{4}+9\left(4 \omega^{2}-A_{1}-B_{1}\right) \lambda_{1}^{2}+A_{1} B_{1}}$,

$g_{4}=\frac{\left(16 \lambda_{1}^{2}-A_{1}\right) \Phi_{4}-8 \omega \lambda_{1} \Phi_{3}}{256 \lambda_{1}^{4}+16\left(4 \omega^{2}-A_{1}-B_{1}\right) \lambda_{1}^{2}+A_{1} B_{1}}$, 
where

$$
\begin{aligned}
\Phi_{0}= & A_{2}+A_{3} g_{1}^{2}, \quad \Phi_{1}=2 A_{2} h_{2}+2 A_{3} g_{1} g_{2}+A_{4} g_{1}^{2}+A_{5}, \\
\Phi_{2}= & B_{2} g_{2}+B_{2} h_{2} g_{1}+B_{3} g_{1}+B_{4} g_{1}^{3}, \\
\Phi_{3}= & A_{2}\left(h_{2}^{2}+2 h_{3}\right)+A_{3}\left(g_{2}^{2}+2 g_{1} g_{3}\right) \\
& +A_{4}\left(h_{2} g_{1}^{2}+2 g_{1} g_{2}\right)+3 A_{5} h_{2}+A_{6}+A_{7} g_{1}^{4}+A_{8} g_{1}^{2}, \\
\Phi_{4}= & B_{2}\left(h_{2} g_{2}+h_{3} g_{1}+g_{3}\right)+B_{3}\left(g_{2}+2 h_{2} g_{1}\right) \\
& +3 B_{4} g_{1}^{2} g_{2}+B_{5} g_{1}^{3}+B_{6} g_{1} .
\end{aligned}
$$

Thus, solution (12) becomes:

$\xi(t)=\sum_{k=1}^{4} h_{k} \mathrm{e}^{k \lambda_{1} t} \epsilon^{k}, \quad \eta(t)=\sum_{k=1}^{4} g_{k} \mathrm{e}^{k \lambda_{1} t} \epsilon^{k}$.

\section{Numerical investigation}

The kind of an asymptotic orbit depends on the sign of the parameter $\epsilon$. This sign together with the linear approximation of (18) provide the possible types of the outgoing and incoming eigenvectors which specify the corresponding asymptotic orbits (Deprit \& Henrard 1965). In particular, these types are determined by the vectors defined by the equilibrium and the points:

$$
\begin{array}{lllll}
\text { I } & : & \xi_{1}=\epsilon \mathrm{e}^{\lambda_{1} t}, & \xi_{2}=\epsilon g_{1} \mathrm{e}^{\lambda_{1} t}, & \text { (out. eigen.) } \\
\text { II }: & \xi_{1}=-\epsilon \mathrm{e}^{\lambda_{1} t}, & \xi_{2}=-\epsilon g_{1} \mathrm{e}^{\lambda_{1} t}, & \text { (out. eigen.) } \\
\text { III }: & \xi_{1}=\epsilon \mathrm{e}^{-\lambda_{1} t}, & \xi_{2}=-\epsilon g_{1} \mathrm{e}^{-\lambda_{1} t}, & \text { (inc. eigen.) } \\
\text { IV }: & \xi_{1}=-\epsilon \mathrm{e}^{-\lambda_{1} t}, & \xi_{2}=\epsilon g_{1} \mathrm{e}^{-\lambda_{1} t} . & \text { (inc. eigen.). }
\end{array}
$$

Thus, non-symmetric doubly asymptotic orbits can be formed by:

(a) : out. eigen. I and inc. eigen. IV,

(b) : out. eigen. II and inc. eigen. III.

while the rest two combinations (I-III and II-IV) give rise to symmetric doubly asymptotic orbits.

In order to locate the pairs of values of the parameters of the problem for which a non-symmetric asymptotic orbit, say of the kind I-IV, exists, we apply a systematic search along a fine grid on the whole plane $(\mu, \omega)$. For any point of this grid, by using (18), we choose the following initial conditions:

$$
\begin{aligned}
& x_{1, \mathrm{I}, 0}=x_{L}+h_{1} \epsilon+h_{2} \epsilon^{2}+h_{3} \epsilon^{3}+h_{4} \epsilon^{4}, \\
& x_{2, \mathrm{I}, 0}=g_{1} \epsilon+g_{2} \epsilon^{2}+g_{3} \epsilon^{3}+g_{4} \epsilon^{4}, \\
& x_{3, \mathrm{I}, 0}=\left(h_{1} \epsilon+2 h_{2} \epsilon^{2}+3 h_{3} \epsilon^{3}+4 h_{4} \epsilon^{4}\right) \lambda_{1}, \\
& x_{4, \mathrm{I}, 0}=\left(g_{1} \epsilon+2 g_{2} \epsilon^{2}+3 g_{3} \epsilon^{3}+4 g_{4} \epsilon^{4}\right) \lambda_{1}, \\
& x_{1, \mathrm{IV}, 0}=x_{L}-h_{1} \epsilon+h_{2} \epsilon^{2}-h_{3} \epsilon^{3}+h_{4} \epsilon^{4}, \\
& x_{2, \mathrm{IV}, 0}=g_{1} \epsilon-g_{2} \epsilon^{2}+g_{3} \epsilon^{3}-g_{4} \epsilon^{4}, \\
& x_{3, \mathrm{IV}, 0}=\left(h_{1} \epsilon-2 h_{2} \epsilon^{2}+3 h_{3} \epsilon^{3}-4 h_{4} \epsilon^{4}\right) \lambda_{1}, \\
& x_{4, \mathrm{IV}, 0}=\left(-g_{1} \epsilon+2 g_{2} \epsilon^{2}-3 g_{3} \epsilon^{3}+4 g_{4} \epsilon^{4}\right) \lambda_{1},
\end{aligned}
$$

along the outgoing and incoming eigenvectors of an equilibrium point and, then, we apply forward and backward integration to the equations of motion (see Perdios 1993). These integrations are continued until the total number of times they intersect the $\mathrm{O} x_{2}$ axis is odd. At the final intersection we will have that:

$x_{1, \mathrm{I}}(\mu, \omega)=x_{1, \mathrm{IV}}(\mu, \omega)=0$.
So, the two integrations form an asymptotic orbit if the following conditions hold at this intersection:

$x_{2, \mathrm{I}}(\mu, \omega)=x_{2, \mathrm{IV}}(\mu, \omega)$,

$x_{4, \mathrm{I}}(\mu, \omega)=x_{4, \mathrm{IV}}(\mu, \omega)$,

while the preservation of the Jacobi integral guarantees that:

$x_{3, \mathrm{I}}(\mu, \omega)=x_{3, \mathrm{IV}}(\mu, \omega)$.

If the conditions (21) are not satisfied within a predetermined mild accuracy, typically $\varepsilon \leq 10^{-2}$, then we ignore this point of the grid and try a neighboring one. In the opposite case, we look for proper corrections of $\mu$ and $\omega$ so that:

$x_{2, \mathrm{I}}(\mu+\delta \mu, \omega+\delta \omega)=x_{2, \mathrm{IV}}(\mu+\delta \mu, \omega+\delta \omega)$
$x_{4, \mathrm{I}}(\mu+\delta \mu, \omega+\delta \omega)=x_{4, \mathrm{IV}}(\mu+\delta \mu, \omega+\delta \omega)$

The corrections $\delta \mu$ and $\delta \omega$ can be approximated by solving the following linearization of (23):

$$
\begin{aligned}
& \left(\frac{\partial x_{2, \mathrm{I}}}{\partial \mu}-\frac{\partial x_{2, \mathrm{IV}}}{\partial \mu}\right) \delta \mu+\left(\frac{\partial x_{2, \mathrm{I}}}{\partial \omega}-\frac{\partial x_{2, \mathrm{IV}}}{\partial \omega}\right) \delta \omega=x_{2, \mathrm{IV}}-x_{2, \mathrm{I}} \\
& \left(\frac{\partial x_{4, \mathrm{I}}}{\partial \mu}-\frac{\partial x_{4, \mathrm{IV}}}{\partial \mu}\right) \delta \mu+\left(\frac{\partial x_{4, \mathrm{I}}}{\partial \omega}-\frac{\partial x_{4, \mathrm{IV}}}{\partial \omega}\right) \delta \omega=x_{4, \mathrm{IV}}-x_{4, \mathrm{I}}
\end{aligned}
$$

To approximate the partial derivatives appearing in the above relations, we have used some additional integrations of the equations of motions, with the same initial conditions and slightly modified values of $\mu$ or $\omega$. The whole procedure is repeated until the conditions (21) are fulfilled within a predefined accuracy (e.g. $10^{-8}$ ). It is obvious that, once a non-symmetric asymptotic orbit of the kind I-IV is found by this procedure, a corresponding solution of the kind II-III is also found. The incoming eigenvector of initial conditions for this solution will be symmetric to the corresponding one of the I-IV orbit with respect to the position of the equilibrium point. The same holds for the outgoing vector of the II-III solution and the outgoing one of the I-IV orbit.

By using this procedure, we have calculated several nonsymmetric asymptotic orbits of the kind I-IV with different combinations of numbers of intersections with the $\mathrm{O} x_{2}$ axis. We restrict our presentation to the results concerning the equilibria $L_{1}, L_{3}$ and the cases of asymptotic orbits 1-2, 1-4 and 16 , i.e. when the forward integration crosses this axis once, while the backward one crosses it twice, four and six times, respectively. The full output of searching for such orbits along the grid on the plane $(\mu, \omega)$ is presented in Fig. 1. The elements of a number of these orbits are given in Tables 1 and 2. In these tables we give the values of $\mu$ and $\omega$ for which these orbits exist, the initial conditions for the forward and backward integrations, the number of times these integrations intersect the $\mathrm{O} x_{2}$ axis as well as the corresponding time of integration. Some of these orbits, labelled by their enumeration in the corresponding table, are shown in Figs. 2 and 3. In some cases, magnifications of the area around the equilibrium point are included in these figures. The curves denoted by a dotted-line represent the corresponding II-III orbits. All these asymptotic orbits are unstable. 
Table 1. Non-symmetric doubly asymptotic orbits at $L_{1}(\epsilon=-0.00001)$.

\begin{tabular}{|c|c|c|c|c|c|c|c|c|}
\hline & $\mu$ & $\omega$ & $\begin{array}{l}x_{1, \mathrm{I}, 0} \\
x_{1, \mathrm{IV}, 0}\end{array}$ & $\begin{array}{l}x_{2, \mathrm{I}, 0} \\
x_{2, \mathrm{IV}, 0}\end{array}$ & $\begin{array}{l}x_{3, \mathrm{I}, 0} \\
x_{3, \mathrm{IV}, 0}\end{array}$ & $\begin{array}{l}x_{4, \mathrm{I}, 0} \\
x_{4, \mathrm{IV}, 0}\end{array}$ & $\begin{array}{l}\mathrm{N}_{\mathrm{I}} \\
\mathrm{N}_{\mathrm{IV}}\end{array}$ & $\begin{array}{l}t_{\mathrm{I}} \\
t_{\mathrm{IV}}\end{array}$ \\
\hline 1 & 0.05840 & 2.55521 & $\begin{array}{l}0.4720124 \\
0.4720324\end{array}$ & $\begin{array}{l}0.0000382 \\
0.0000382\end{array}$ & $\begin{array}{l}-0.0000099 \\
-0.0000099\end{array}$ & $\begin{array}{r}0.0000378 \\
-0.0000378\end{array}$ & $\begin{array}{l}1 \\
2\end{array}$ & $\begin{array}{r}19.2616665 \\
-17.1332105\end{array}$ \\
\hline 2 & 0.07364 & 2.37942 & $\begin{array}{l}0.4764450 \\
0.4764650\end{array}$ & $\begin{array}{l}0.0000251 \\
0.0000251\end{array}$ & $\begin{array}{l}-0.0000138 \\
-0.0000138\end{array}$ & $\begin{array}{r}0.0000347 \\
-0.0000347\end{array}$ & $\begin{array}{l}1 \\
2\end{array}$ & $\begin{array}{r}9.9273726 \\
-15.0305573\end{array}$ \\
\hline 3 & 0.03800 & 2.04208 & $\begin{array}{l}0.5684306 \\
0.5684506\end{array}$ & $\begin{array}{l}0.0000209 \\
0.0000209\end{array}$ & $\begin{array}{l}-0.0000141 \\
-0.0000141\end{array}$ & $\begin{array}{r}0.0000295 \\
-0.0000295\end{array}$ & $\begin{array}{l}1 \\
2\end{array}$ & $\begin{array}{l}28.4335362 \\
-9.8693437\end{array}$ \\
\hline 4 & 0.10808 & 2.58120 & $\begin{array}{l}0.4159246 \\
0.4159446\end{array}$ & & & & $\begin{array}{l}1 \\
4\end{array}$ & $\begin{array}{r}10.3126852 \\
-13.5570871\end{array}$ \\
\hline 5 & 0.11614 & 2.11987 & & & & & $\begin{array}{l}1 \\
4\end{array}$ & $\begin{array}{r}5.2806554 \\
-13.2903478\end{array}$ \\
\hline 6 & 0.10752 & 1.97391 & $\begin{array}{l}0.4853007 \\
0.4853207\end{array}$ & & & $\begin{array}{r}0.0000278 \\
-0.0000278\end{array}$ & $\begin{array}{l}1 \\
4\end{array}$ & $\begin{array}{r}4.9525071 \\
-11.1761201\end{array}$ \\
\hline 7 & 0.00809 & 1.54476 & $\begin{array}{l}0.7252355 \\
0.7252555\end{array}$ & & & $\begin{array}{r}0.0000222 \\
-0.0000222\end{array}$ & $\begin{array}{l}1 \\
4\end{array}$ & $\begin{array}{r}10.4573533 \\
-45.4606286\end{array}$ \\
\hline 8 & 0.02378 & 1.97388 & & & & $\begin{array}{r}0.0000 \\
-0.0000\end{array}$ & $\begin{array}{l}1 \\
4\end{array}$ & $\begin{array}{r}16.8353927 \\
-17.3771796\end{array}$ \\
\hline 9 & 0.04713 & 1.79739 & & & & & $\begin{array}{l}1 \\
4\end{array}$ & $\begin{array}{r}6.0297037 \\
-14.8441368\end{array}$ \\
\hline 10 & 0.24837 & 2.64511 & $\begin{array}{l}0.2627185 \\
0.2627385\end{array}$ & $\begin{array}{l}0.0000269 \\
0.0000269\end{array}$ & $\begin{array}{l}-0.0000143 \\
-0.0000143\end{array}$ & $\begin{array}{r}0.0000 \\
-0.0000\end{array}$ & $\begin{array}{l}1 \\
4\end{array}$ & $\begin{array}{r}8.5375160 \\
-11.2404381\end{array}$ \\
\hline 11 & 0.01662 & 1.69524 & $\begin{array}{l}0.6691123 \\
0.6691323\end{array}$ & $\begin{array}{l}0.0000181 \\
0.0000181\end{array}$ & $\begin{array}{l}-0.0000134 \\
-0.0000134\end{array}$ & $\begin{array}{r}0.0000243 \\
-0.0000243\end{array}$ & $\begin{array}{l}1 \\
4\end{array}$ & $\begin{array}{r}9.1207637 \\
-29.8966029\end{array}$ \\
\hline 12 & 0.32798 & 2.39447 & $\begin{array}{l}0.1901608 \\
0.1901808\end{array}$ & $\begin{array}{l}0.0000153 \\
0.0000153\end{array}$ & $\begin{array}{l}-0.0000223 \\
-0.0000223\end{array}$ & $\begin{array}{r}0.0000340 \\
-0.0000340\end{array}$ & $\begin{array}{l}1 \\
4\end{array}$ & $\begin{array}{r}5.2416553 \\
-9.9744977\end{array}$ \\
\hline 13 & 0.04687 & 2.13716 & $\begin{array}{l}0.5417077 \\
0.5417277\end{array}$ & $\begin{array}{l}0.0000216 \\
0.0000216\end{array}$ & $\begin{array}{l}-0.0000143 \\
-0.0000143\end{array}$ & $\begin{array}{r}0.0000309 \\
-0.0000309\end{array}$ & $\begin{array}{l}1 \\
6\end{array}$ & $\begin{array}{r}10.9308880 \\
-21.9435338\end{array}$ \\
\hline 14 & 0.08551 & 2.11081 & $\begin{array}{l}0.4963779 \\
0.4963979\end{array}$ & $\begin{array}{l}0.0000164 \\
0.0000164\end{array}$ & $\begin{array}{l}-0.0000184 \\
-0.0000184\end{array}$ & $\begin{array}{r}0.0000301 \\
-0.0000301\end{array}$ & $\begin{array}{l}1 \\
6\end{array}$ & $\begin{array}{r}6.5519783 \\
-15.5179660\end{array}$ \\
\hline 15 & 0.03142 & 1.84721 & & $\begin{array}{l}0.0000174 \\
0.0000174\end{array}$ & $\begin{array}{l}-0.0000152 \\
-0.0000152\end{array}$ & $\begin{array}{r}0.0000264 \\
-0.0000264\end{array}$ & $\begin{array}{l}1 \\
6\end{array}$ & $\begin{array}{r}8.0141364 \\
-25.8794205\end{array}$ \\
\hline 16 & 0.41232 & 2.39349 & $\begin{array}{l}0.0968582 \\
0.0968782 \\
\end{array}$ & $\begin{array}{l}0.0000146 \\
0.0000146 \\
\end{array}$ & $\begin{array}{l}-0.0000232 \\
-0.0000232 \\
\end{array}$ & $\begin{array}{r}0.0000339 \\
-0.0000339 \\
\end{array}$ & $\begin{array}{l}1 \\
6 \\
\end{array}$ & $\begin{array}{r}3.9637354 \\
-15.5200498 \\
\end{array}$ \\
\hline
\end{tabular}

Table 2. Non-symmetric doubly asymptotic orbits at $L_{3}(\epsilon=-0.00001)$.

\begin{tabular}{|c|c|c|c|c|c|c|c|c|}
\hline & $\mu$ & $\omega$ & $\begin{array}{l}x_{1, \mathrm{I}, 0} \\
x_{1, \mathrm{IV}, 0}\end{array}$ & $\begin{array}{l}x_{2, \mathrm{I}, 0} \\
x_{2, \mathrm{IV}, 0}\end{array}$ & $\begin{array}{l}x_{3, \mathrm{I}, 0} \\
x_{3, \mathrm{IV}, 0}\end{array}$ & $\begin{array}{l}x_{4, \mathrm{I}, 0} \\
x_{4, \mathrm{IV}, 0}\end{array}$ & $\begin{array}{l}\mathrm{N}_{\mathrm{I}} \\
\mathrm{N}_{\text {IV }}\end{array}$ & $\begin{array}{l}t_{\mathrm{I}} \\
t_{\mathrm{IV}}\end{array}$ \\
\hline 1 & 0.03304 & 1.19099 & $\begin{array}{l}-0.9044402 \\
-0.9044202\end{array}$ & $\begin{array}{l}0.0000475 \\
0.0000475\end{array}$ & $\begin{array}{l}-0.0000037 \\
-0.0000037\end{array}$ & $\begin{array}{r}0.0000177 \\
-0.0000177\end{array}$ & $\begin{array}{l}1 \\
2\end{array}$ & $\begin{array}{r}28.5196613 \\
-33.9079200\end{array}$ \\
\hline 2 & 0.03162 & 1.16583 & $\begin{array}{l}-0.9165003 \\
-0.9164803\end{array}$ & $\begin{array}{l}0.0000490 \\
0.0000490\end{array}$ & $\begin{array}{l}-0.0000035 \\
-0.0000035\end{array}$ & $\begin{array}{r}0.0000173 \\
-0.0000173\end{array}$ & $\begin{array}{l}1 \\
2\end{array}$ & $\begin{array}{r}29.9466110 \\
-40.2602246\end{array}$ \\
\hline 3 & 0.01120 & 1.40272 & $\begin{array}{l}-0.8031180 \\
-0.8030980\end{array}$ & $\begin{array}{l}0.0000766 \\
0.0000766\end{array}$ & $\begin{array}{l}-0.0000027 \\
-0.0000027\end{array}$ & $\begin{array}{r}0.0000210 \\
-0.0000210\end{array}$ & $\begin{array}{l}1 \\
4\end{array}$ & $\begin{array}{r}36.2269541 \\
-50.8251018\end{array}$ \\
\hline 4 & 0.12442 & 1.06805 & $\begin{array}{l}-1.0098229 \\
-1.0098029\end{array}$ & $\begin{array}{l}0.0000254 \\
0.0000254\end{array}$ & $\begin{array}{l}-0.0000061 \\
-0.0000061\end{array}$ & $\begin{array}{r}0.0000156 \\
-0.0000156\end{array}$ & $\begin{array}{l}1 \\
4\end{array}$ & $\begin{array}{r}19.0135315 \\
-32.5214012\end{array}$ \\
\hline 5 & 0.02943 & 1.34491 & $\begin{array}{l}-0.8339802 \\
-0.8339602\end{array}$ & $\begin{array}{l}0.0000480 \\
0.0000480\end{array}$ & $\begin{array}{l}-0.0000042 \\
-0.0000042\end{array}$ & $\begin{array}{r}0.0000200 \\
-0.0000200\end{array}$ & $\begin{array}{l}1 \\
4\end{array}$ & $\begin{array}{r}25.2833982 \\
-40.9746666\end{array}$ \\
\hline 6 & 0.02526 & 1.35656 & $\begin{array}{l}-0.8274196 \\
-0.8273996\end{array}$ & $\begin{array}{l}0.0000517 \\
0.0000517\end{array}$ & $\begin{array}{l}-0.0000039 \\
-0.0000039\end{array}$ & $\begin{array}{r}0.0000202 \\
-0.0000202\end{array}$ & $\begin{array}{l}1 \\
6\end{array}$ & $\begin{array}{r}26.6734260 \\
-46.9066897\end{array}$ \\
\hline 7 & 0.03044 & 1.33529 & $\begin{array}{l}-0.8383500 \\
-0.8383300\end{array}$ & $\begin{array}{l}0.0000474 \\
0.0000474\end{array}$ & $\begin{array}{l}-0.0000042 \\
-0.0000042\end{array}$ & $\begin{array}{r}0.0000198 \\
-0.0000198\end{array}$ & $\begin{array}{l}1 \\
6\end{array}$ & $\begin{array}{r}25.1706379 \\
-45.5030715\end{array}$ \\
\hline 8 & 0.04541 & 1.28393 & $\begin{array}{l}-0.8667431 \\
-0.8667231\end{array}$ & $\begin{array}{l}0.0000394 \\
0.0000394\end{array}$ & $\begin{array}{l}-0.0000048 \\
-0.0000048\end{array}$ & $\begin{array}{r}0.0000190 \\
-0.0000190\end{array}$ & $\begin{array}{l}1 \\
6\end{array}$ & $\begin{array}{r}22.4257795 \\
-42.5493786\end{array}$ \\
\hline 9 & 0.07125 & 1.10274 & $\begin{array}{l}-0.9673826 \\
-0.9673626\end{array}$ & $\begin{array}{l}0.0000333 \\
0.0000333\end{array}$ & $\begin{array}{l}-0.0000049 \\
-0.0000049\end{array}$ & $\begin{array}{r}0.0000162 \\
-0.0000162\end{array}$ & $\begin{array}{l}1 \\
6\end{array}$ & $\begin{array}{r}21.3559251 \\
-60.5284322\end{array}$ \\
\hline 10 & 0.09859 & 1.01689 & $\begin{array}{l}-1.0301337 \\
-1.0301137\end{array}$ & $\begin{array}{l}0.0000292 \\
0.0000292\end{array}$ & $\begin{array}{l}-0.0000051 \\
-0.0000051\end{array}$ & $\begin{array}{r}0.0000149 \\
-0.0000149\end{array}$ & $\begin{array}{l}1 \\
6\end{array}$ & $\begin{array}{r}22.4216992 \\
-52.7887116\end{array}$ \\
\hline 11 & 0.00747 & 1.39120 & $\begin{array}{l}-0.8058180 \\
-0.8057980\end{array}$ & $\begin{array}{l}0.0000941 \\
0.0000941\end{array}$ & $\begin{array}{l}-0.0000022 \\
-0.0000022\end{array}$ & $\begin{array}{r}0.0000208 \\
-0.0000208\end{array}$ & $\begin{array}{l}1 \\
6\end{array}$ & $\begin{array}{r}43.7679904 \\
-76.1293972\end{array}$ \\
\hline 12 & 0.00899 & 1.22547 & $\begin{array}{l}-0.8771886 \\
-0.8771686\end{array}$ & $\begin{array}{l}0.0000901 \\
0.0000901\end{array}$ & $\begin{array}{l}-0.0000020 \\
-0.0000020\end{array}$ & $\begin{array}{r}0.0000183 \\
-0.0000183\end{array}$ & $\begin{array}{l}1 \\
6\end{array}$ & $\begin{array}{r}48.2669857 \\
-74.8791219\end{array}$ \\
\hline 13 & 0.05441 & 1.17521 & $\begin{array}{l}-0.9216531 \\
-0.9216331\end{array}$ & $\begin{array}{l}0.0000372 \\
0.0000372\end{array}$ & $\begin{array}{l}-0.0000047 \\
-0.0000047\end{array}$ & $\begin{array}{r}0.0000174 \\
-0.0000174\end{array}$ & $\begin{array}{l}1 \\
6\end{array}$ & $\begin{array}{r}23.5028343 \\
-49.7589320\end{array}$ \\
\hline
\end{tabular}



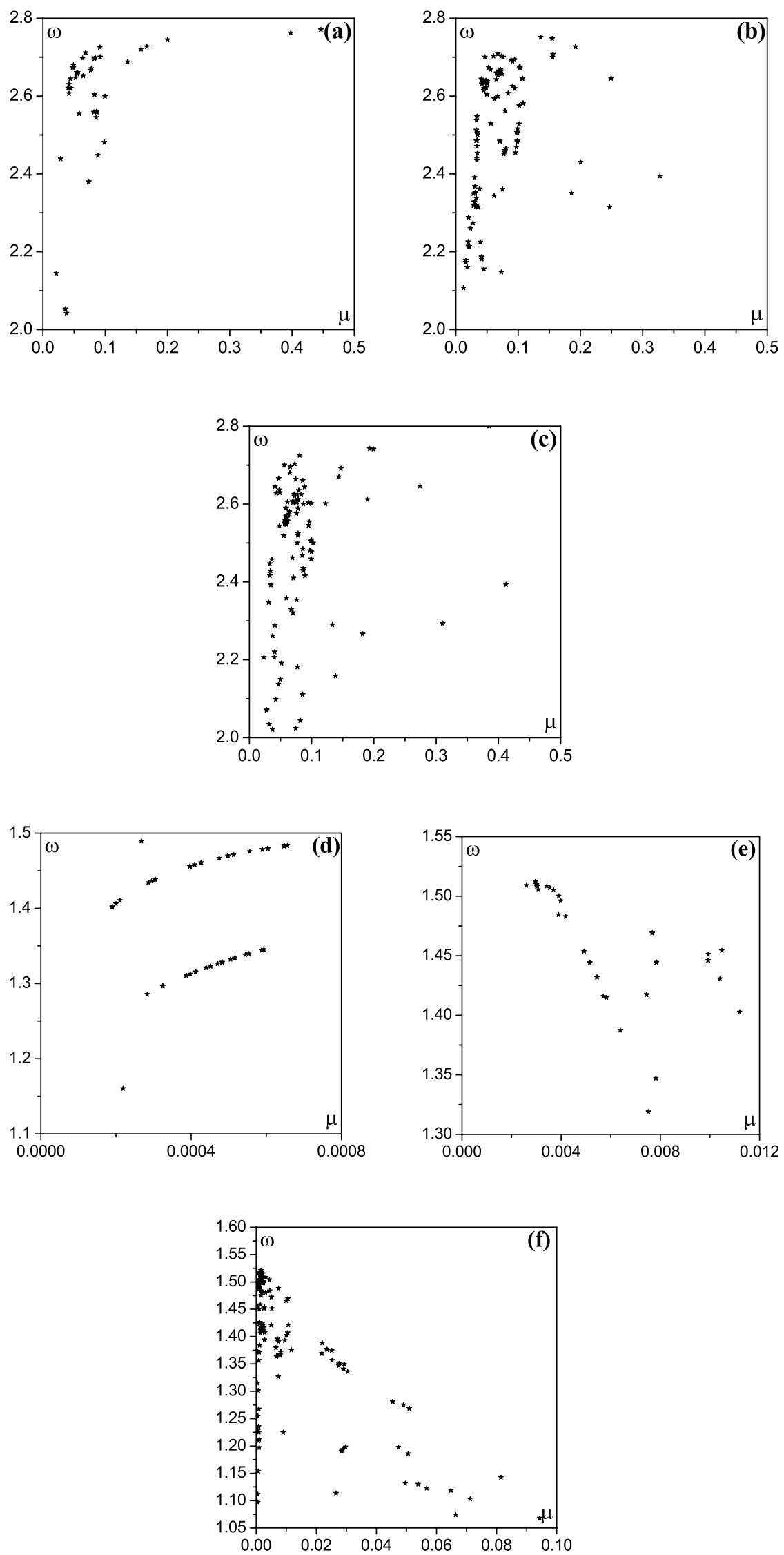

Fig. 1. Pairs of values of $(\mu, \omega)$ for which I-IV and II-III non-symmetric asymptotic orbits at $L_{1}$ with numbers of intersections a) 1-2, b) 1-4 and c) 1-6, have been found to exist. Figures d), e) and f) present the corresponding results for $L_{3}$. 

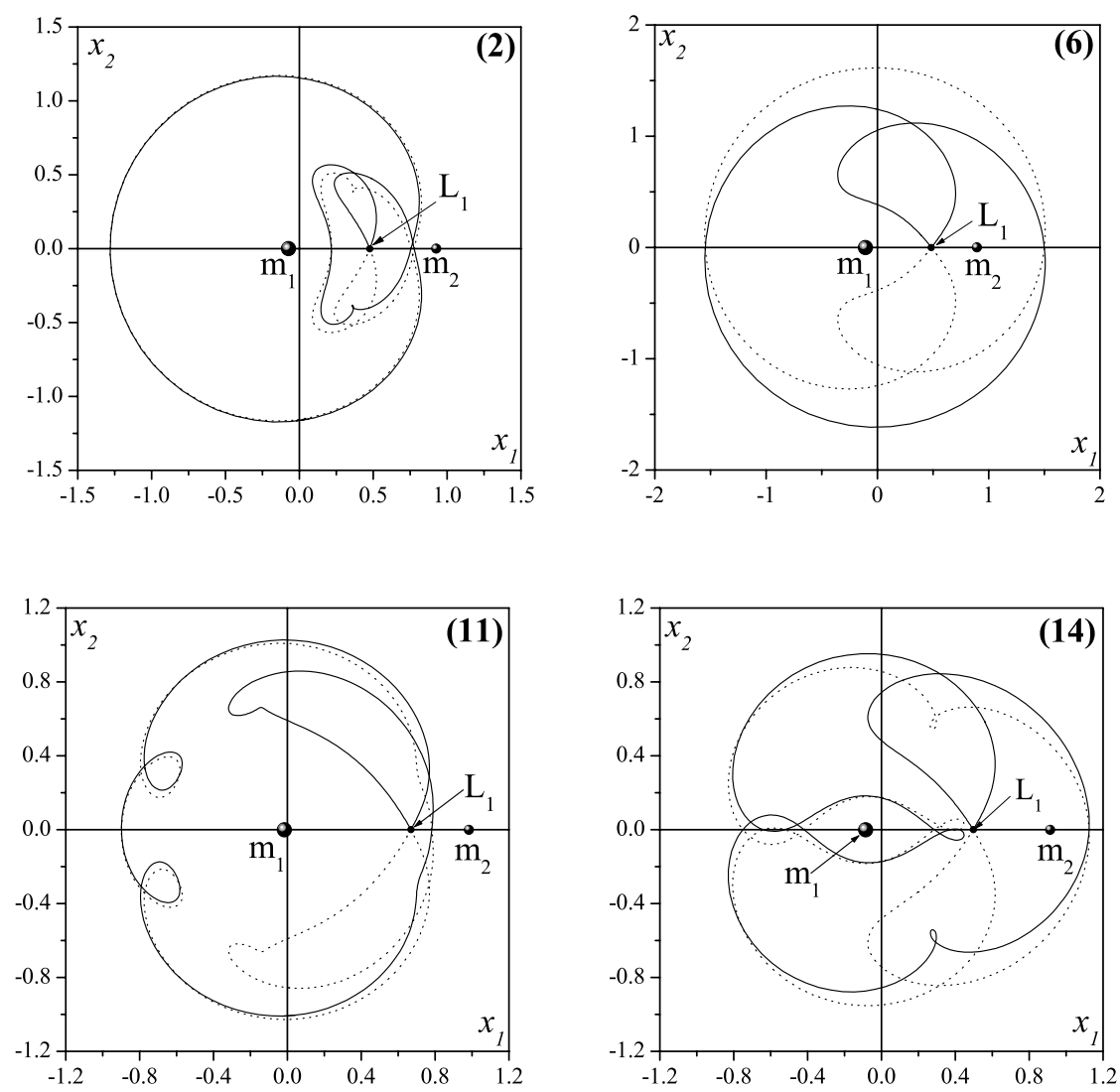

Fig. 2. Non-symmetric doubly asymptotic orbits at $L_{1}$. The orbits are labelled by their enumeration in Table 1 . The curves denoted by a continuous line denote orbits of kind I-IV while the dotted-lines represent the corresponding II-III orbits.

The abovementioned results have been obtained by using a suitably small value for the orbital parameter $\epsilon$ equal to $=-0.00001$. The choice of this value is based on the accuracy required for $\mu$ and $\omega$. In practice $\epsilon$ is chosen by the criterion that a backward integration of the equations of motion by reversing the signs of the velocity coordinates in the initial conditions (19), should recapture the corresponding equilibrium point within a predetermined accuracy. The abovementioned value of $\epsilon$ guaranties that the values of $\mu$ and $\omega$ will be correct up to five fractional figures. We have also verified that the reverse integrations recapture the the libration points within an accuracy of at least five significant figures.

Asymptotic motion of the abovementioned kind is strongly connected to periodic motion around the collinear equilibria. More precisely, for each pair of the I-IV and II-III orbits already presented, there is a neighboring symmetric periodic orbit which is surrounded by them. This orbit belongs to the Liapunov family related to the corresponding equilibrium point. Consider, for example, the orbits of kinds I-IV and II-III that are determined by the elements of the sixth entry of Table 1. In order to compute the symmetric periodic solution extracted by these orbits, we have to apply very slight modifications to the initial conditions $x_{10}=x_{1, \mathrm{I}}, x_{40}=x_{4, \mathrm{I}}$, while $x_{20}=x_{30}=0$. These modifications can be calculated by using a classical corrector scheme (see, for example, Ragos \& Zagouras 1991). The elements of the obtained periodic orbit are $x_{10}=0.48503714, x_{20}=x_{30}=0, x_{40}=0.00001721$, $T / 2=15.44804931$. We have also calculated the family which
Table 3. The family $f_{L_{1}}$ : a family consisting of $\mathrm{O} x_{1}$-symmetric periodic orbits and generated from the sixth asymptotic orbit of Table 1 $(\mu=0.10752, \omega=1.97391)$.

\begin{tabular}{ccccc}
\hline \hline$x_{10}$ & $x_{40}$ & $x_{1, T / 2}$ & $C$ & $T / 2$ \\
\hline 0.57392350 & -0.48061821 & 0.57455443 & -4.34680646 & 23.15005900 \\
0.48530714 & 0.00001721 & 0.48555686 & -4.45672900 & 15.44804931 \\
0.57382005 & -0.48032263 & 0.57427847 & -4.34680646 & 22.75713408 \\
\hline
\end{tabular}

contains this orbit. We name this family $f_{L_{1}}$. Figures $4 \mathrm{a}$ and $4 \mathrm{~b}$ exhibit the characteristic curves $\left(T / 2, x_{01}\right)$ and $\left(T / 2, x_{04}\right)$ of $f_{L_{1}}$. This family terminates at both ends asymptotically to a simple periodic solution around $L_{1}$ and spiraling asymptotic to $L_{4}$. This occurs since the eigenvalues of matrix $\mathcal{M}$ at $L_{4}$ and $L_{5}$ are, in this case, complex $( \pm 0.51568795 \pm i 2.83874257)$. Figures $4 c$ and $4 \mathrm{~d}$ present two orbits near the two ends of the family, while the corresponding data are given in the first and the last entries of Table 3. Magnifications of the area around $L_{1}$ are given in Figs. 4c-1 and 4d-1, respectively.

Following the same procedure, we have also calculated a symmetric periodic orbit by using the data given in the third entry of Table 2. The elements of this orbit are $x_{10}=-0.8031199$, $x_{20}=x_{30}=0, x_{40}=0.00001028, T / 2=63.27570908$. Figures $5 \mathrm{a}$ and $5 \mathrm{~b}$ present the characteristic curves $\left(T / 2, x_{01}\right)$ and $\left(T / 2, x_{04}\right)$ of the family. The family that contains this orbit is denoted by $f_{L_{3}}$. This family leads to collision with the less massive primary and terminate asymptotically at both ends to a 

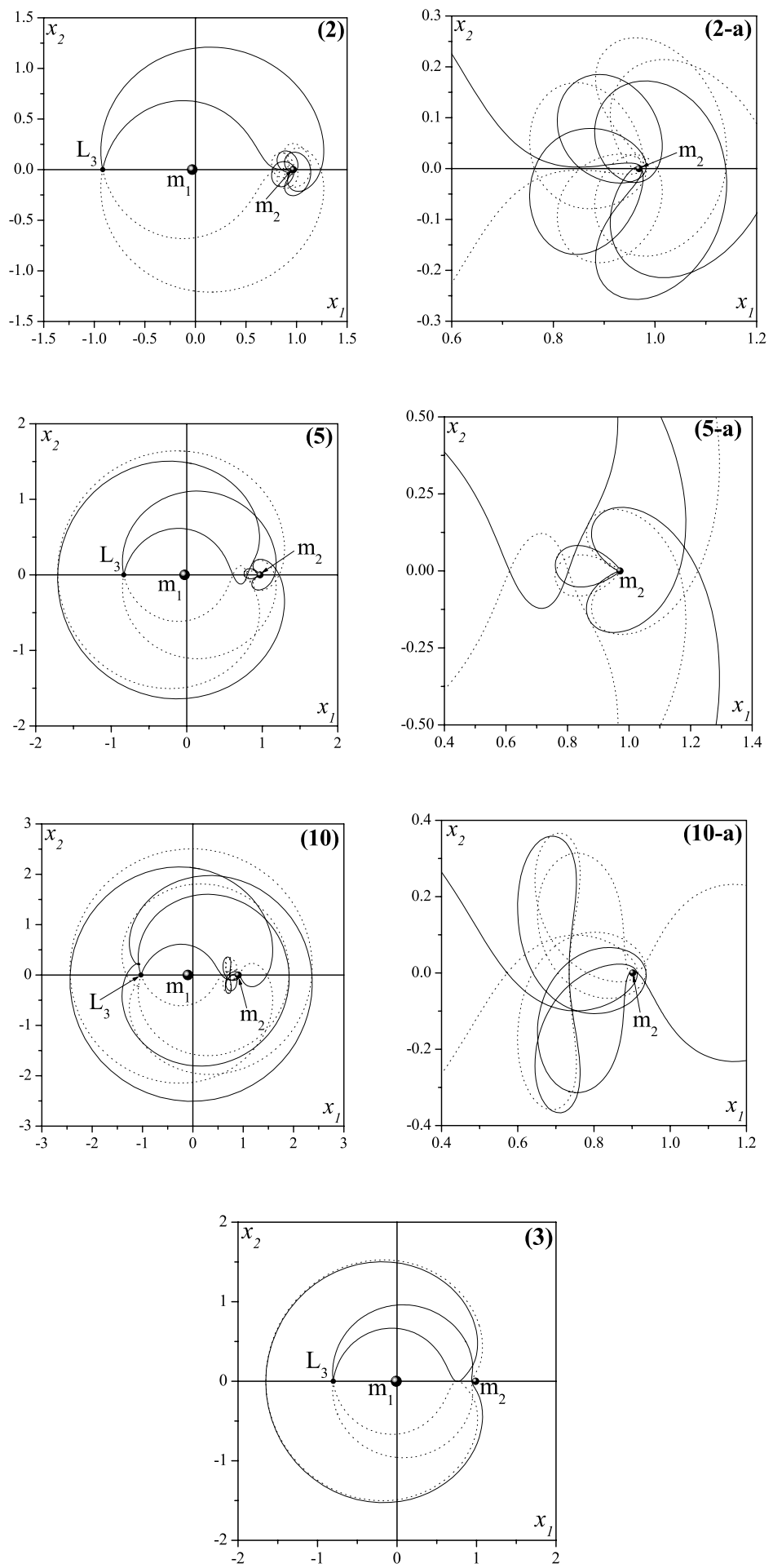

Fig. 3. Non-symmetric doubly asymptotic orbits at $L_{3}$. The orbits are labelled by their enumeration in Table 2 . In most cases, magnifications of the area around the equilibrium point are included. The curves denoted by a continuous line denote orbits of kind I-IV while the dotted-lines represent the corresponding II-III orbits.

simple periodic solution around $L_{3}$. Figures $5 \mathrm{c}$ and $5 \mathrm{~d}$ exhibit two such orbits, respective magnifications of the area around $L_{1}$ are given in Figs. 5c-1 and 5d-1. The relative data are given in the first and the last entries of Table 4 . All members of $f_{L_{1}}$ and $f_{L_{3}}$ are unstable.

The elements of the simple periodic solution at which $f_{L_{1}}$ terminates asymptotically are $x_{10}=0.57393156$, $x_{20}=x_{30}=0, x_{40}=0.48064123, T / 2=1.30826785 . \mathrm{A}$ graphical representation of this solution is given in Fig. 4e. Some members of the family containing this orbit are exhibited in Fig. 4f. It is obvious that this family is analogous to the family $c$ of the classical restricted problem. Similarly, the initial conditions and half-period of the simple periodic solution at which $f_{L_{3}}$ terminates asymptotically are $x_{10}=-0.76142281$, 

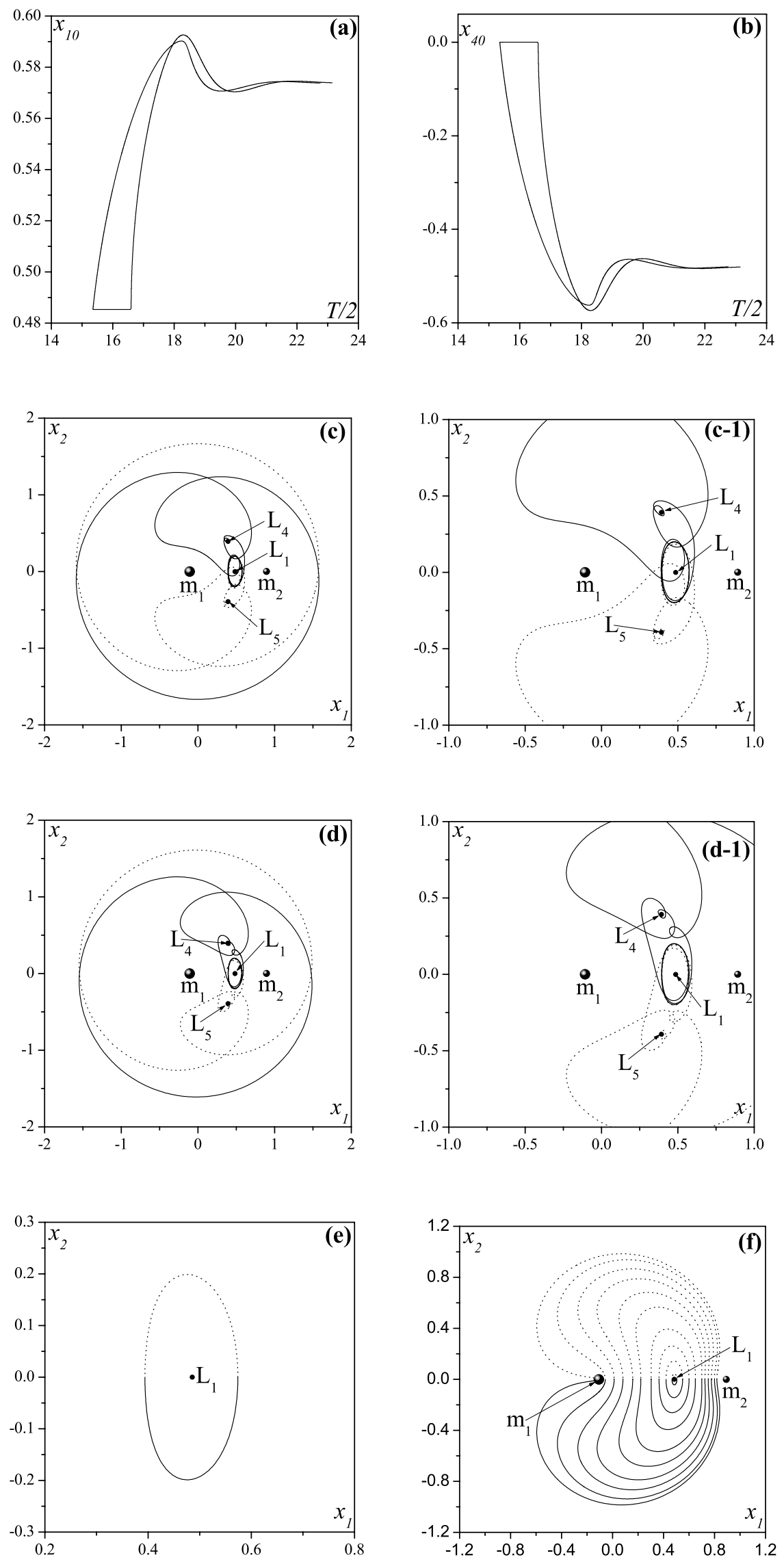

Fig. 4. a)-b) The characteristic curves $\left(T / 2, x_{01}\right)$ and $\left(T / 2, x_{04}\right)$ of family $f_{L_{1}}(\mu=0.10751, \omega=1.97390)$. c)-d) Two orbits at the ends of $f_{L_{1}}$. -1 -1)-d-1) Magnifications of the previous figures in the vicinity of $L_{1}$. e) The simple symmetric periodic solution to which $f_{L_{1}}$ terminates asymptotically. f) Some members of the family that contains this solution. 

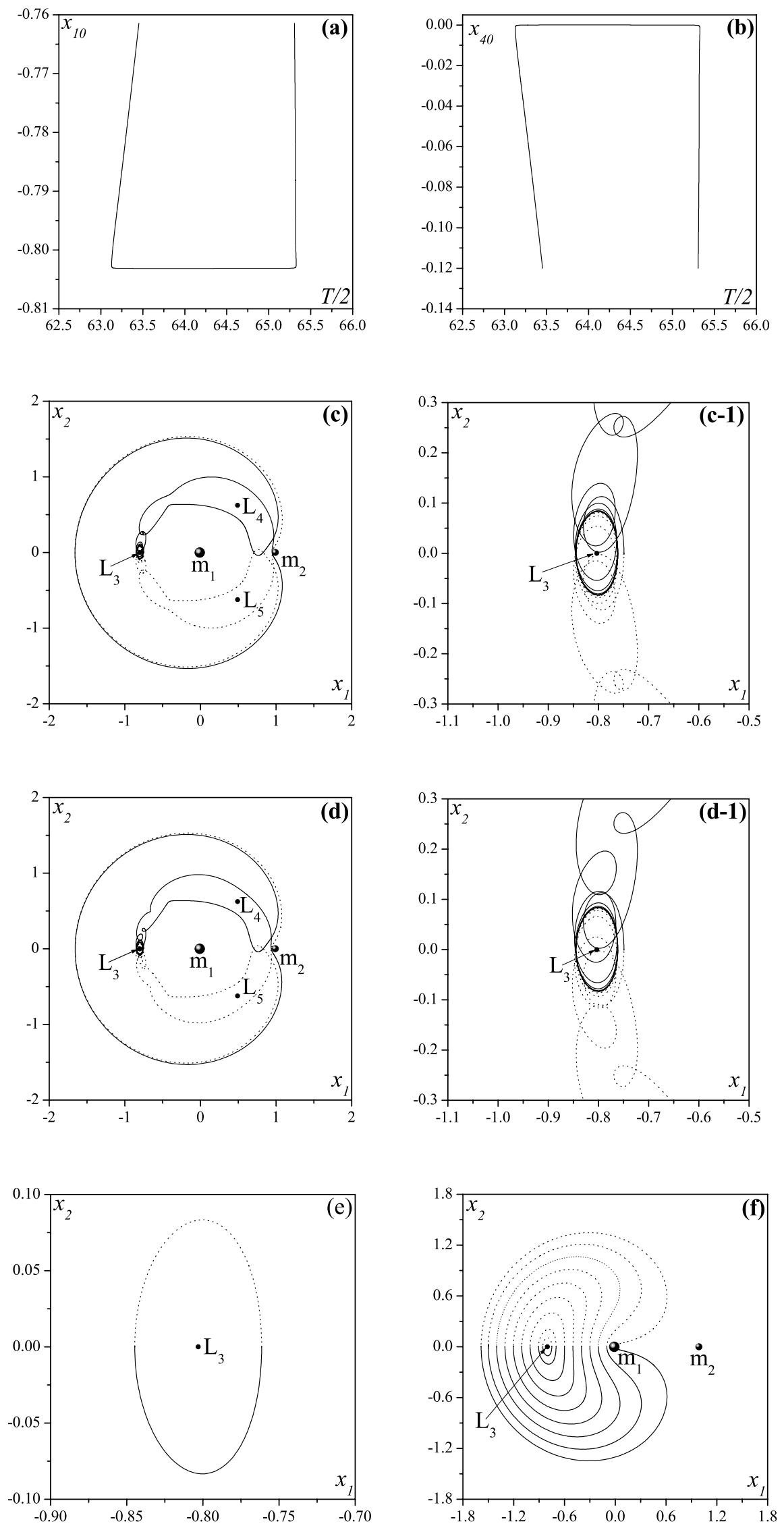

Fig. 5. a)-b) The characteristic curves $\left(T / 2, x_{01}\right)$ and $\left(T / 2, x_{04}\right)$ of family $f_{L_{3}}(\mu=0.01120, \omega=1.40271)$. c)-d $)$ Two orbits at the ends of $f_{L_{1}}$. c-1)-d-1) Magnifications of the previous figures in the vicinity of $L_{3}$. e) The simple symmetric periodic solution to which $f_{L_{3}}$ terminates asymptotically. f) Some members of the family that contains this solution. 
Table 4. The family $f_{L_{3}}$ : a family consisting of $\mathrm{O} x_{1}$-symmetric periodic orbits and generated from the third asymptotic orbit of Table 2 $(\mu=0.01120, \omega=1.40272)$.

\begin{tabular}{ccccc}
\hline \hline$x_{10}$ & $x_{40}$ & $x_{1, T / 2}$ & $C$ & $T / 2$ \\
\hline-0.76143594 & -0.12002404 & -0.74921174 & -3.77514423 & 65.30610995 \\
-0.80311990 & 0.00001028 & -0.78264483 & -3.77882610 & 63.27570908 \\
-0.76143918 & 0.12002404 & -0.74920475 & -3.77514253 & 63.45304649 \\
\hline
\end{tabular}

$x_{20}=x_{30}=0, x_{40}=-0.12005274, T / 2=2.21204419$. This orbit along with some members of the corresponding family are presented in Figs. 5e and 5f. This family is congruent to the family $b$ of the classical restricted problem. All these solutions are also unstable.

\section{References}

Chermnykh, S. V. 1987, Vest. Leningrad Univ., 2(8), 10

Danby, J. M. A. 1967, AJ, 72, 198

Deprit, A., \& Henrard, J. 1965, AJ, 70, 271
Gożdziewski, K., \& Maciejewski, A. J. 1998, Celest. Mech. Dynam. Astron., 70, 41

Koon, W. S., Lo, M. W., Marsden, J. E., \& Ross, S. D. 2000, Chaos, 10(2), 427

Llibre, J., Martinez, R., \& Simó, C. 1985, J. Diff. Eqs, 58, 104

Markellos, V. V. 1991, in Predictability, stability and chaos in N-body dynamical systems, ed. A. E. Roy (New York: Plenum Press), 413

Perdios, E. 1983, in Dynamical Trapping and Evolution in the Solar System, ed. V. V. Markellos, \& Y. Kozai (D. Reidel Publ. Co.), 339

Perdios, E. A., \& Markellos, V. V. 1990, Ap\&SS, 166, 129

Perdios, E. A. 1993, Ap\&SS, 199, 185

Perdios, E. A. 1996, Ap\&SS, 240, 141

Perdios, E. A. 1997, Ap\&SS, 254, 61

Perdios, E. A., Ragos, O., Perdiou, A. E., \& Vrahatis, M. N. 2001, Nonlinear Anal., 199, 185

Perdios, E. A., \& Kalantonis, V. S. 2002, A\&A, 394, 323

Ragos, O., \& Zagouras, C. 1991, Celest. Mech. Dynam. Astron., 50, 325

Strömgren, E. 1935, Copenhagen Obs. Publ., No. 100 